OPEN ACCESS

Edited by:

Magnus Hook,

Texas A\&M Health Science Center,

United States

Reviewed by:

Thomas Proft,

The University of Auckland,

New Zealand

Marco Rinaldo Oggioni,

University of Leicester,

United Kingdom

*Correspondence:

Markus Hilleringmann

markus.hilleringmann@hm.edu

Specialty section:

This article was submitted to Infectious Diseases,

a section of the journal

Frontiers in Microbiology

Received: 10 October 2020

Accepted: 20 January 2021

Published: 09 February 2021

Citation:

Ness $S$ and Hilleringmann $M$ (2021) Streptococcus pneumoniae Type 1 Pilus - A Multifunctional Tool

for Optimized Host Interaction.

Front. Microbiol. 12:615924. doi: 10.3389/fmicb.2021.615924

\section{Streptococcus pneumoniae Type 1 Pilus - A Multifunctional Tool for Optimized Host Interaction}

\author{
Stefan Ness and Markus Hilleringmann* \\ FG Protein Biochemistry \& Cellular Microbiology, Department of Applied Sciences and Mechatronics, Munich University \\ of Applied Sciences, Munich, Germany
}

Streptococcus pneumoniae represents a major Gram-positive human pathogen causing bacterial pneumonia, otitis media, meningitis, and other invasive diseases. Several pneumococcal isolates show increasing resistance rates against antibacterial agents. A variety of virulence factors promote pneumococcal pathogenicity with varying importance in different stages of host infection. Virulence related hair-like structures ("pili") are complex, surface located protein arrays supporting proper host interaction. In the last two decades different types of pneumococcal pili have been identified: pilus-1 (P1) and pilus-2 (P2) are formed by the catalytic activity of sortases that covalently assemble secreted polypeptide pilin subunits in a defined order and finally anchor the resulting pilus in the peptidoglycan. Within the long pilus fiber the presence of intramolecular isopeptide bonds confer high stability to the sequentially arranged individual pilins. This mini review will focus on S. pneumoniae TIGR4 P1 molecular architecture, the subunits it builds and provides insights into P1 sortasemediated assembly. The complex P1 architecture (anchor-/backbone-/tip-subunits) allows the specific interaction with various target structures facilitating different steps of colonization, invasion and spreading within the host. Optimized pilin subunit confirmation supports P1 function under physiological conditions. Finally, aspects of P1- host interplay are summarized, including recent insights into P1 mechanobiology, which have important implications for $\mathrm{P} 1$ mediated pathogenesis.

\footnotetext{
Keywords: pilus, bacteria, Streptococcus pneumoniae, architecture, sortase, virulence, host-interaction, mechanobiology
}

\section{INTRODUCTION: Streptococcus pneumoniae A MAJOR HUMAN PATHOGEN EXPRESSING DIFFERENT TYPES OF PILI}

Streptococcus pneumoniae (the pneumococcus) is a human commensal bacterium that can cause lethal diseases like pneumonia, septicemia, and meningitis. As major human pathogen, it provokes high morbidity and mortality rates especially in children and the elderly. Licensed pneumococcal vaccines are not covering all relevant virulent strains and an increasing number of antibiotic resistant isolates makes treatment challenging (Subramanian et al., 2019). Novel, broad-spectrum vaccination strategies and new antibacterials are of utmost importance to combat S. pneumoniae. 
The switch from a human commensal to an invasive pneumococcal pathogen and its disease causing capacity in various host niches is an area of intense study (Loughran et al., 2019). A multiplicity of differentially regulated cell surface located molecules mediate the complex interplay of S. pneumoniae and the human host. This includes several types of high molecular weight protein assemblies, so-called pili, which promote pneumococcal virulence. Although S. pneumoniae was first isolated by Pasteur in 1881, pneumococcal pili were discovered only at the beginning of the 21st century. Besides a recently described type IV competence pilus (Laurenceau et al., 2013; Muschiol et al., 2019), pneumococcal isolates express two variants of Gram-positive, sortase cross-linked multi-subunit pili ["pilus-1 (P1) and pilus-2 (P2)"] (Barocchi et al., 2006; Bagnoli et al., 2008). In this review, we focus on the assembly and particular architecture of pneumococcal P1 and derived from that summarize its role as multifunctional host-interaction tool. This indicates a virulence-mediating role of P1 at different phases of pneumococcal diseases and an optimized P1-structure for various host environments.

\section{SUBUNITS AND SORTASE-MEDIATED ASSEMBLY OF PNEUMOCOCCAL PILUS-1}

While typical Gram-negative pili are formed by non-covalent interactions between pilins, the covalent assembly of Grampositive pili is catalyzed by specific sortases involving pilus subunit polymerization and cell wall anchoring of the resulting pilus fiber (Telford et al., 2006; Hendrickx et al., 2011; Hospenthal et al., 2017). Streptococcus pneumoniae TIGR4, belonging to the highly invasive pneumococcal serotype 4 , is a patient isolate were P1 was initially identified (Barocchi et al., 2006) that represents an important reference strain studying P1 biology. Major components involved in pneumococcal TIGR4 $\mathrm{P} 1$ formation are clustered in a defined genetic region [pilus island 1 (PI-1)] that encodes 3 P1 specific class C sortases (SrtC1, SrtC-2, SrtC-3), 3 P1 subunits (RrgA, RrgB, and RrgC) and a transcriptional regulator (RlrA) (Figure 1-A1). Analysis of strain collections indicates that only a subset of pneumococcal isolates expresses P1 ( 30\%) (Dzaraly et al., 2020). Electron microscopic analysis allowed the initial visualization of pili on the surface of negative stained TIGR4 isolates. Negative staining procedure improves contrast for better visualization of few $\mathrm{nm}$ thin pilus filaments. The explicit identification of PI-1 encoded pilins within $\mathrm{P} 1$ required specific immuno-labeling strategies and resulted in heterotrimeric P1 working models with $\operatorname{RrgB}$ as major pilin and 2 minor pilins (RrgA and RrgC) (Barocchi et al., 2006; LeMieux et al., 2006, 2008; Hilleringmann et al., 2008; Fälker et al., 2008).

Similar to other Gram-positive pilus systems, the current model of P1 formation involves pilus subunit- and Srtsynthesis in the cytoplasm and respective surface localization followed by a biphasic process of pilus-polymerization and anchoring to the bacterial cell wall (Khare and Narayana, 2017; Figure 1-A2): After their cytoplasmic expression, respective
$\mathrm{N}$-terminal signal sequences of P1-pilins and -sortases allow Sec-translocation into the exoplasmic zone. A C-terminal hydrophobic stretch functions as membrane anchor and promotes their embedding into the lipid bilayer. Since P1 discovery, a main research focus lies on the respective role of PI-1 sortases for P1 assembly, including molecular details of the catalyzed transpeptidation and Srt-regulation: during P1 polymerization, PI-1 individual class $\mathrm{C}$ sortases recognize particular variants of LPXTG motif pentapeptide-cell wall sorting signals (CWSSs) at the C-terminus of individual P1 pilins as described for other Gram-positive pilus systems. The sortasecatalytic activity hydrolyses between the CWSS threonine and glycine residues by an initial nucleophilic attack of the threonine's carbonyl carbon atom via the enzyme's active site cysteine residue (Figure 1-A2/I). The Thr C-terminus in the formed sortase-pilin thioacyl intermediate is linked to a conserved lysine $\varepsilon$-amino group within the pilin motif of the next P1 subunit of the growing pilus, $\operatorname{RrgB}$ K183 (within RrgB pilin motif WXXXVXVYPK) and finally specific lysine residue of RrgC (structural analysis suggest Lys K142 as respective nucleophilic residue of $\mathrm{RrgC}$, although experimental proof is missing; (Figure 1-A2/II-III; LeMieux et al., 2006, 2008; Fälker et al., 2008; Manzano et al., 2008; Neiers et al., 2009a,b; El Mortaji et al., 2012b; Shaik et al., 2014). The data support a gradual selectivity of the three PI-1 SrtC isoforms to individual P1 pilin CWSSs during P1 polymerization (Figure 1A2/II). Differences in CWSSs (RrgA: YPRTG; RrgB: IPQTG; RrgC: VPDTG) and resulting conformational changes might direct binding preferences of respective PI-1 SrtCs. Recent molecular dynamics simulations suggest a key role of the middle amino acid in the five-residue pilin CWSSs for selective and specific sorting signal targeting of the individual SrtCs (Naziga and Wereszczynski, 2017).

The second step in P1 biosynthesis is the covalent linkage of the polymerized P1 to the bacterial cell wall. Deletion of minor pilin RrgC leads to P1 polymers secreted into the supernatant and identifies RrgC as P1 cell wall anchor molecule (Hilleringmann et al., 2009; Shaik et al., 2014). The primary sortase for Lipid II-mediated RrgC anchoring to peptidoglycan is the pneumococcal housekeeping sortase SrtA (Shaik et al., 2014; Naziga and Wereszczynski, 2017). This is in agreement with SrtA pilus-anchoring activity in other Gram-positive pili systems.

Both, PI-1 class C-Srts and SrtA are central elements for P1 formation. Their coordinated activity seems essential for proper P1 assembly. Interestingly, all P1 class C Srts contain a $\mathrm{N}$-terminal lid-region that covers the active catalytic site triad region constituted of His, Arg, and Cys that is missing in the respective housekeeping sortase and suggest a regulatory element of SrtCs activity. Whereas initial results indicate a flexible, "mobile" lid behavior of SrtC-1 in solution (Manzano et al., 2009), recent data propose a rather rigid SrtC-lid condition in the absence of substrate with a potential negative regulatory function (Jacobitz et al., 2016).

Some important questions regarding the mechanistics of Srt-mediated P1 fiber assembly are ambiguous: (i) Precise chronology of P1 formation including the factors determining the starting of the biosynthesis until final anchoring of P1 


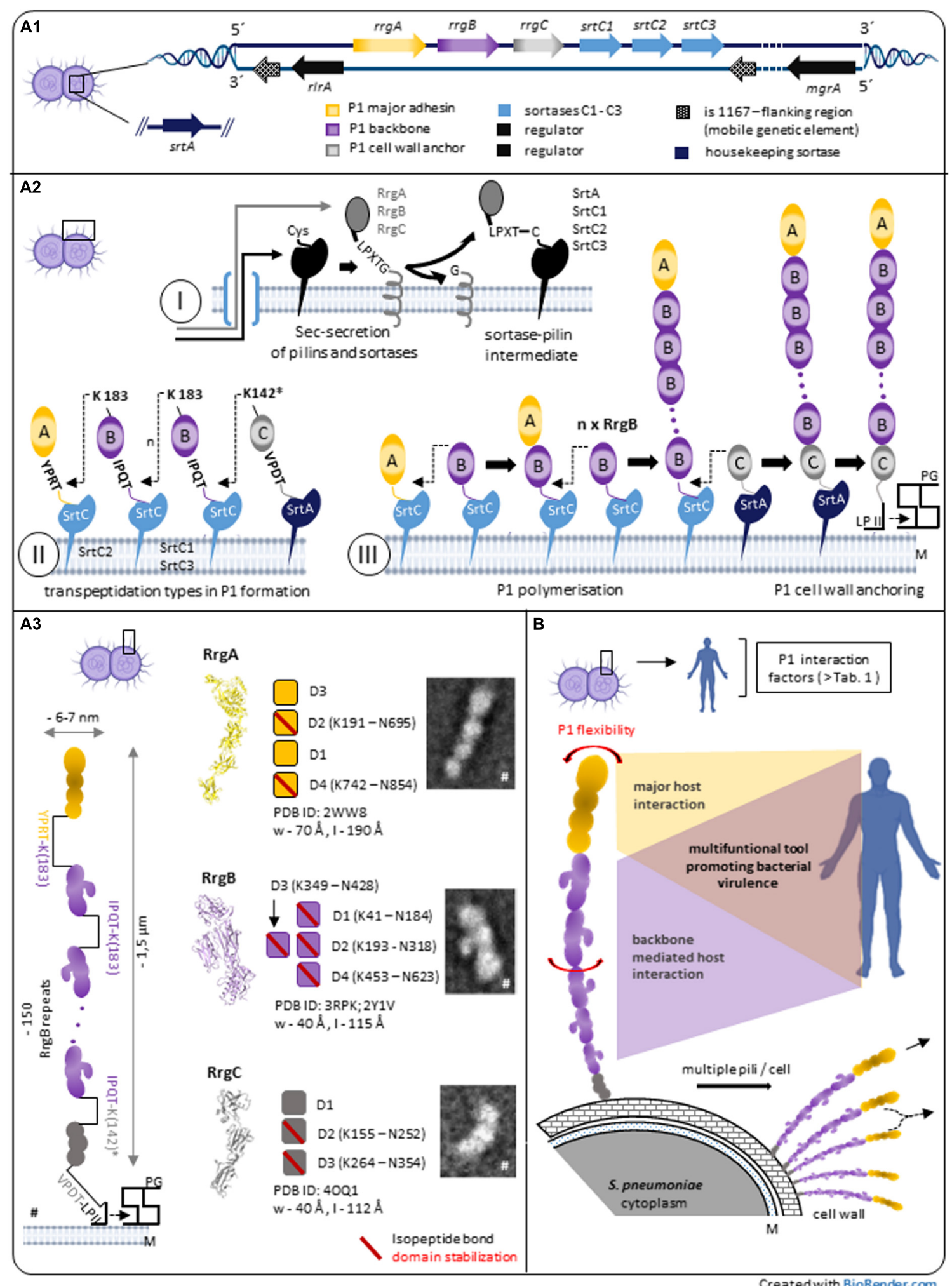

FIGURE 1 | Pilus-1 of Streptococcus pneumoniae - assembly, architecture and structure-derived properties. (A1) Streptococcus pneumoniae TIGR4 pilus-island 1 (PI-1) and related genes involved in P1 biosynthesis. (A2) Model of sortase-mediated assembly of pneumococcal pilus-1. A2-l: P1-pilins and relevant sortases are expressed in the cytoplasm, exported by the Sec secretion system and surface located via a C-terminal hydrophobic stretch in the bacterial plasma membrane. The initial step of sortase-catalyzed transpeptidation is a pilin CWSS specific hydrolysis between Thr and Gly of particular variants of the LPXTG motif via the enzyme's 


\begin{abstract}
FIGURE 1 | Continued
active site Cys resulting in a sortase-pilin thioacyl intermediate. A2-Il: C-terminal pilin Thr in the activated sortase-pilin complex (A2-I) is linked to a conserved Lys in the next P1 subunit of the growing pilus. P1 heterotrimeric formation is a complex process involving the sequential incorporation of RrgA, RrgB, and RrgC via pilin specific Pl-1 class C-sortases. Shown are details of individual P1 sortase-pilin intermediates and respective Lys residues involved in transpeptidation reactions during P1 formation. Simulation results combined with existing biochemical and genetic data of several groups suggest the following PI-1 class C-Srt specificities (Naziga and Wereszczynski, 2017): SrtC1 is the primary Srt for RgB polymerization; in the absence of RrgB, SrtC1 might add RrgA, and RrgC to the pilus. SrtC3 shows a pronounced RrgB crosslinking activity when compared to RrgA and RrgC although less distinctive than SrtC1-RrgB. The first substrate of SrtC2 is RrgA linking the $\mathrm{P} 1$ adhesin to the RrgB backbone. Apart from background activity of SrtC-1, housekeeping SrtA is defined as principal RrgC-related sortase. A2-III: P1 assembly is a biphasic process of P1 polymerization and final, lipid II-mediated, cell wall anchoring. (A3) P1 heterotrimeric architecture and pilin characteristics. P1 is a thin, long filament composed of a proximal RrgC anchor, $n$-serial RrgB backbone molecules and a distal RrgA adhesin. TEM images and high-resolution structures of P1-pilins (RrgA, RrgB, and RrgC) identify elongated molecules containing intramolecular, isopeptide bond stabilized domains. A distinctive feature of RrgB is a lateral subunit (D3) that is involved in host interaction (" parts adapted from Hilleringmann et al., 2009). (B) P1 expressing pneumococci exhibit multiple pilus filaments on the bacterial surface functioning as single fiber or potential P1 aggregations. In addition to P1 subunit stabilization (A3), the entire P1 fiber shows a pronounced bendability. Longitudinal rotation of serial arranged RrgB subunits along the P1 axis suggest additional optimization of P1 architecture. P1 host interaction is complex, mainly mediated by terminal adhesin RrgA. Force induced RrgB host factor interaction indicate a functional role of P1 backbone in addition to its role as stabilized stalk exposing terminal P1 adhesin. *Potential nucleophilic RrgC Lys residue. CWSS, cell wall sorting signal; LP II, lipid II; M, bacterial plasma membrane; PG, peptidoglycan; P1, Pilus-1; Srt, Sortase.
\end{abstract}

to the cell wall is missing. (ii) Sequence details of P1 pilin incorporation: although major backbone $\operatorname{RrgB}$ polymerization was observed in a RrgA deletion strain background (Nelson et al., 2007), the "tip first rule" proposing a first transpeptidation reaction between the terminal pilus adhesin and a pilin backbone molecule [as described for the well studied heterotrimeric SpaA pili of Corynebacterium diphtheriae (Ramirez et al., 2020)] seems reasonable for other Gram-positive pili systems, but needs detailed experimental proof in the P1 system. (iii) Regulation of P1 length: based on a model of "pilus chain terminator role" of anchor pilin SpaB in C. diphtheriae SpaA pilus (Mandlik et al., 2008), it should be investigated whether the step of $\mathrm{RrgC}$ anchor incorporation into the SrtC-activated $\operatorname{RrgB}_{n} \operatorname{RrgA}$ fiber has a similar role in defining P1 final length. Importantly proper regulation of P1 RrgB mediated length determines the relative position of major adhesin RrgA and might be crucial for P1 functionality as recently demonstrated in other Gram-positive pilus systems (Chang et al., 2019).

TIGR4 bacterial surface is covered with multiple copies of P1 (Barocchi et al., 2006; Hilleringmann et al., 2009). The regulation of the spatio-temporal P1 distribution, including signal sequence directed cellular export and surface-positioning of pilins and Srts (Spirig et al., 2011), plus the precise interplay between P1 assembly and peptidoglycan metabolism is not understood. Topological studies describe a specific pattern of P1 subunits on the bacterial surface ("symmetric P1 foci") indicating a nonhomogenous P1 distribution (Fälker et al., 2008).

In addition to specific control mechanisms focused on proper P1 biosynthesis, signals from general regulatory networks of the complex interplay between $S$. pneumoniae and the human host are supposed to influence P1 expression (Kreikemeyer et al., 2011; Gómez-Mejia et al., 2018). Transcriptional P1 regulation seems to be of major importance: besides a suggested negative regulation by RrgA (Basset et al., 2011), PI-1 was described to be positively controlled by a RlrA feedback loop (Basset et al., 2012). In addition to central $r l r A$, other PI-1 positive regulators were described (Hava et al., 2003; Kreikemeyer et al., 2011). Within the growing list of negative regulators (Basset et al., 2011; Kreikemeyer et al., 2011; Herbert et al.,
2015), some of them might require additional validation (Basset et al., 2017). Detailed investigations with extending systems that reflect the in vivo situation as closely as possible will help to further clarify the overall context of pneumococcal P1 regulation during host colonization and invasion (Pancotto et al., 2013; Figueira et al., 2014). The described absence of $\mathrm{P} 1$ expression below $31^{\circ} \mathrm{C}$ indicates the importance of host environmental factors on $\mathrm{P} 1$ regulation (Basset et al., 2017). Analysis of PI-1 containing isolates identified subpopulations of P1-expressing and - non expressing bacteria. Angelis et al. (2011), Basset et al. (2011) which might be a compromise balancing P1-related advantages like improved human colonization and -potential disadvantages of P1 induced immunogenicity and biosynthesis costs (Binsker et al., 2020; Iovino et al., 2020).

\section{DESIGN OF MULTIMERIC TIGR4 PILUS-1 ARCHITECTURE REFLECTS AN OPTIMIZED INTERACTION TOOL}

High resolution analysis of surface digested individual pili allowed a detailed analysis of heterotrimeric P1 architecture and pilin stoichiometry, overcoming interferences and reducing complexity of respective cell bound-P1 study objects. TIGR4 P1 basic structure is a long, only $\sim 6-7 \mathrm{~nm}$-wide filament composed of a multiple repetition of $\mathrm{RrgB}$ backbone molecules, with one RrgA adhesin and one RrgC minor pilin at the P1 distal and proximal end, respectively, associated in a head-totail covalently linked fashion. Scanning TEM analysis indicated that a $1.5-\mu \mathrm{m}$-long pilus comprises approximately $150 \mathrm{RrgB}$ monomers in which a nose-like protrusion in each $\operatorname{RrgB}$ subunit defines the polarity of the fiber (Hilleringmann et al., 2009; Figure 1-A3). Adjacent P1 filaments on the cell wall surface can tangle or form bundles. Aggregations of different length pili with terminal RrgA, could explain the described P1 coiled-coiled phenotype with surface located RrgA molecules (Hilleringmann et al., 2008). RrgA aggregates were described as better TLR2 agonist in vitro when compared to RrgA monomer (Basset et al., 2013). Respectively, clustered P1 RrgA on the bacterial surface 
might be better recognized by the innate immune receptor TLR2 in vivo.

High resolution crystal structures of all P1-pilins have been resolved (Figure 1-A3): major adhesion RrgA (PDB: 2WW8) represents a four domain, elongated molecule, carrying segments of eukaryotic origin important for host interaction, mainly mediated by RrgA D3 domain (Izoré et al., 2010; Moschioni et al., 2010). The backbone RrgB (PDB: 3RPK and 2Y1V) subunit displays a four domain fold (Paterson and Baker, 2011; El Mortaji et al., 2012a). Interestingly a domain (D3) is arranged laterally of the $\operatorname{RrgB}$ middle domain (D2) which constitutes a special structural feature among Gram-positive major pilins (Young et al., 2014) and presumes a specific P1 adaptation for optimized interaction (Becke et al., 2019). Reconstruction of P1 architecture by fitting of the P1 fiber with high resolution crystal structure of $\operatorname{RrgB} \mathrm{D} 2-\mathrm{D} 4$ molecules and RrgB D1 computer model data showed a rotation along the longitudinal P1 axis between two neighboring $\operatorname{RrgB}$ subunits of about $17-22^{\circ}$ (Spraggon et al., 2010). A resulting different spatial domain arrangement of sequential RrgB molecules might further improve host interaction via multiple linearly arranged RrgB molecules (Figure 1-B), like a RrgB D3 mediated collagen interaction (Becke et al., 2019). Anchor subunit $\operatorname{RrgC}$ is the smallest P1 pilin and folds into three independent domains (PDB: 4OQ1) (Shaik et al., 2014). P1 pilins show a characteristic domain-based architecture that incorporated into P1 filament resemble a "beads on a string" macroscopic phenotype. Like described for other Gram-positive pilus systems, the existence of eukaryotic IgG-like subdomain variants within all P1 pilins (Izoré et al., 2010; Paterson and Baker, 2011; Shaik et al., 2014) suggest an adaptation strategy for proper P1 mediated host factor interaction while minimizing adverse host immune system responses by mimicry of eukaryotic immune system elements (Krishnan et al., 2007; Shaik et al., 2014).

Structural analysis of Gram-positive pilins revealed domain stabilization via intramolecular isopeptide bond formations (Kang et al., 2007; Baker et al., 2015). P1 subunits show different numbers of stabilized individual domains [RrgA: 2 (Izoré et al., 2010); RrgB: 4 (El Mortaji et al., 2010, 2012a; Paterson and Baker, 2011), and RrgC: 2 (Shaik et al., 2014); Figure 1-A3] that might reflect individual pilin adaptation to generate an optimized P1 multimeric tool able to withstand mechanical perturbations induced during host interaction and supporting P1 assembly. Recent single-molecule force spectroscopy data propose structural concepts to protect covalent bonds of Grampositive pilins from cleavage under mechanical challenge (Echelman et al., 2016).

In addition to pure pilus mechanical stability and similar to other Gram-positive pili, functional P1 mediated host interaction requires a certain extent of flexibility of the P1-filament to efficiently target host structures (Figure 1-B). Macroscopic analysis of P1 phenotype of cell-bound and isolated pili revealed a substantial degree of fiber bendability (Barocchi et al., 2006; Hilleringmann et al., 2009). The respective percental contribution of the flexible linker regions between individual P1 pilins within the fiber and subunit derived flexibility, i.e., interdomain malleability, needs further investigation and might be P1 subunit specific (Spraggon et al., 2010; El Mortaji et al., 2012a).

\section{MULTIFUNCTIONAL PNEUMOCOCCAL TYPE-1 PILI PROMOTE VARIOUS INTERACTION SCENARIOS}

The deletion of $\mathrm{P} 1$ attenuated the virulence of respective clinical isolates in mouse models of pneumococcal infection (Barocchi et al., 2006). Work by several groups indicate a significant involvement of major adhesin RrgA in various steps of pneumococcal colonization and virulence addressing different host niches, which is described in a recent, comprehensive review (Iovino et al., 2020) and summarized in Table 1A). RrgA mediates in a $\operatorname{RrgB}$ backbone independent way, adherence to host epithelial cells, potentially promoting initial host colonization (Nelson et al., 2007). Dose-dependent interactions of RrgA with ECM molecules (fibronectin, laminin, and collagen I), not seen for $\operatorname{RrgB}$ and $\operatorname{RrgC}$, indicate specific binding to host cell associated molecules (Hilleringmann et al., 2008). In addition to static ELISA approaches, data from single molecule force spectroscopy specify a two-domain binding mechanism for RrgA with Fn, that suggest a P1-mediated bacterial adaptation to keep contact with host tissue surface in shear force environments (Becke et al., 2018). RrgA was found to interact with complement receptor 3 (CR3) that promotes CR3 -mediated uptake of S. pneumoniae expressing P1-RrgA by macrophages and results in pneumococcal stimulation of macrophage motility. Additionally, expression of P1 RrgA together with host expression of CR3 affects virulence and spreading of the pathogen from local sites to the bloodstream in mice (Orrskog et al., 2012). Data of a RrgA D3 dependent TLR2activation and induction of inflammatory TNF- $\alpha$ responses (Basset et al., 2013) suggest further P1-mediated interaction with components of the innate immune system. Overall implications of the RrgA-mediated TLR2-activation of the host immune response, a potential limiting consequence of P1 expression on pneumococcal fitness and virulence, need further investigation. The described more potent activation of TLR2 by oligmeric forms of RrgA might be mediated by aggregated monomeric RrgA of individual P1 fibers on the pneumococcal surface (Figure 1-B). Lethal meningitis caused by $S$. pneumoniae requires the penetration of the blood-brain-barrier (BBB) by the bacteria (Iovino et al., 2016b): an interaction of $\operatorname{RrgA}$ with polymeric immunoglobulin receptor (pIgR) and platelet endothelial cell adhesion molecule 1 (PECAM-1), two BBB endothelial receptors, was found to promote the entry of bacteria into the brain and meningitis development (Iovino et al., 2017). Interestingly, a subpopulation of spherical single pneumococci, that do not express DivIVA, with surfacelocated P1-RrgA seem to be favored to cross the BBB. In addition to the expression of $\operatorname{RrgA}$, the small size of these pneumococcal variants might explain easier penetration of the BBB (Iovino et al., 2016a). In addition, recent data imply that meningitis related neuronal death is mediated by an interaction of $\operatorname{RrgA}$ and pneumolysin with $\beta$-actin 
TABLE 1 | Streptococcus pneumoniae pilus-1 mediated interactions.

\begin{tabular}{|c|c|c|c|c|}
\hline Pilin (PI-1) & Variant & Category* & Major characteristics and target & References \\
\hline \multirow[t]{10}{*}{ A: RrgA_PBD: 2WW8 } & $\begin{array}{l}\text { RrgA recombinant; bacteria } \\
\text { associated RrgA (P1-variants) }\end{array}$ & 1 & $\begin{array}{l}\text { RrgA binds to human respiratory epithelial A594 cells } \\
\text { (independent of P1-RrgB polymerization); P1-Rrg A } \\
\text { mediates colonization of the upper respiratory tract in mice. }\end{array}$ & Nelson et al., 2007 \\
\hline & $\begin{array}{l}\text { recombinant RrgA; purified P1 } \\
\text { (TIGR4 WT) }\end{array}$ & 1 & $\begin{array}{l}\text { Dose-dependent binding of RrgA monomer and isolated pili } \\
\text { to ECM components (fibronectin, collagen I, laminin)/ELISA. }\end{array}$ & Hilleringmann et al., 2008 \\
\hline & $\begin{array}{l}\text { recombinant RrgA FL and } \\
\text { individual RrgA domains }\end{array}$ & $1^{\circ}$ & $\begin{array}{l}\text { D3/D4 - domain binding mechanism of RrgA with ECM } \\
\text { fibronectin under force/AFM-based single molecule force } \\
\text { spectroscopy. }\end{array}$ & Becke et al., 2018 \\
\hline & $\begin{array}{l}\text { RrgA recombinant; bacteria } \\
\text { associated RrgA (P1-variants) }\end{array}$ & $|/| \mid$ & $\begin{array}{l}\text { RrgA mediated binding to } \mathrm{CR} 3 \text { and } \mathrm{CR} 3 \text { dependent uptake } \\
\text { of RrgA containing pneumococci by macrophages; } \\
\text { RrgA-expression promotes systemic pneumococcal spread } \\
\text { and virulent in mice expressing CR3. }\end{array}$ & Orrskog et al., 2012 \\
\hline & $\begin{array}{l}\text { RrgA recombinant; bacteria } \\
\text { associated RrgA (P1-variants) }\end{array}$ & 1 & $\begin{array}{l}\text { P1 functions as TLR2 agonist - with major contribution of } \\
\text { RrgA D3 - in human epithelial cells. Rekombinant RrgA } \\
\text { oligomers show increased TLR } 2 \text { activation. RrgA D3 is } \\
\text { involved in pneumococcal mediated TLR2-activation, } \\
\text { TNF- } \alpha \text { induction and virulence in a mouse model of } \\
\text { infection. }\end{array}$ & Basset et al., 2013 \\
\hline & $\begin{array}{l}\text { RrgA recombinant; bacteria } \\
\text { associated RrgA (P1-variants) }\end{array}$ & $\mid / I I I$ & $\begin{array}{l}\text { RrgA-containing P1 facilitate passage of S. pneumoniae } \\
\text { through the blood-brain barrier (BBB) to cause lethal } \\
\text { meningitis. Favored variants passing BBB of mice are } \\
\text { spherical, single, P1-RrgA + pneumococci. }\end{array}$ & lovino et al., 2016a \\
\hline & & & $\begin{array}{l}\text { Binding of RrgA to BBB endothelial receptors (PECAM-1 } \\
\text { and plgR) promotes bacterial entry and meningitis } \\
\text { development. }\end{array}$ & lovino et al., 2017 \\
\hline & & & $\begin{array}{l}\text { Interaction of RrgA and pneumolysin with } \beta \text {-actin stimulate } \\
\text { meningitis related neuronal death. }\end{array}$ & Tabusi et al., 2020 \\
\hline & $\begin{array}{l}\text { Bacteria associated RrgA } \\
\text { (P1-variants) }\end{array}$ & I + IV & $\begin{array}{l}\text { Potential role of RrgA in biofilm formation / promotion of } \\
\text { inter-bacterial interaction. }\end{array}$ & Muñoz-Elías et al., 2008 \\
\hline & $\begin{array}{l}\text { RrgA recombinant; bacteria } \\
\text { associated RrgA (P1-variants) }\end{array}$ & $\mathrm{I}+\mathrm{n} / \mathrm{a}$ & $\begin{array}{l}\text { Role of RrgA as lectin targeting different host glycosylation } \\
\text { pattern. }\end{array}$ & Day et al., 2017 \\
\hline \multirow[t]{2}{*}{ B: Rrg B_PDB: 3RPK/2Y1V } & $\begin{array}{l}\text { RrgB recombinant; bacteria } \\
\text { P1-variants }\end{array}$ & $\mathrm{I}+\mathrm{n} / \mathrm{a}$ & $\begin{array}{l}\text { Role of RrgB as lectin targeting different host glycosylation } \\
\text { pattern. }\end{array}$ & Day et al., 2017 \\
\hline & $\begin{array}{l}\text { Recombinant RrgB FL and } \\
\text { RrgB } \Delta \text { D3 variants }\end{array}$ & $1^{\circ}$ & $\begin{array}{l}\text { RrgB binds to ECM collagen I in a force-dependent manner } \\
\text { and depends on the orientation of lateral RrgB D3-domain } \\
\text { and the respective position of the collagen fibrils. }\end{array}$ & (Becke et al., 2019 \\
\hline C: RrgC_PDB: 4OQ1 & $\begin{array}{l}\text { RrgC recombinant; bacteria } \\
\text { P1-variants }\end{array}$ & $\mathrm{I}+\mathrm{n} / \mathrm{a}$ & $\begin{array}{l}\text { Role of RrgC as lectin targeting different host glycosylation } \\
\text { pattern. }\end{array}$ & Day et al., 2017 \\
\hline
\end{tabular}

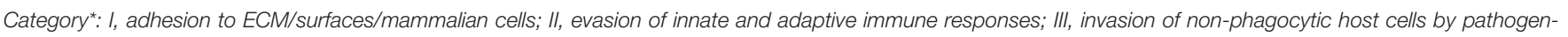
directed endocytosis; $N$, cell-cell aggregation during biofilm formation.

'Interaction under mechanical force conditions; n/a, not available.

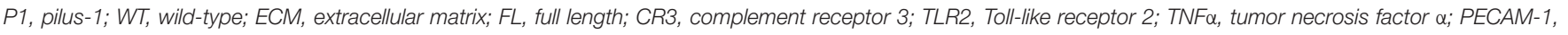
platelet endothelial cell adhesion molecule 1; plgR, polymeric immunoglobulin receptor; AFM, atomic force microscopy.

of human neurons (Tabusi et al., 2020). The formation of biofilms promotes the persistence of pathogenic bacteria on patient's tissues and overall virulence (Muhammad et al., 2020). In vitro screening for biofilm-altered TIGR4 mutants identified RrgA as potential molecule favoring bacterial interaction (Muñoz-Elías et al., 2008).

Polymerized backbone subunits of Gram-positive pili are primarily considered as stabilized and flexible stalk exposing tip based adhesins for proper host interaction. Recent data suggest a specific role for backbone pilins in host interaction, as shown for pili of Streptococcus pyogenes (Tsai et al., 2017; Chen et al., 2020). Interestingly, under mechanical load, P1 RrgB was found to strongly interact with human collagen I (Table 1B) not measurable using static ELISA (Hilleringmann et al., 2008;
Becke et al., 2019). The particular lateral domain D3 of RrgB (Figure 1-A3) is essential for the observed exceptionally high binding forces and a force-induced bond strengthening, a described property of bacterial adhesins (Herman-Bausier et al., 2018). This implies a discrete functional role of the $P 1$ backbone in host factor interaction. Specific binding characteristics mediated by numerous linearly arranged RrgB molecules within P1 needs further investigation. Broader experimental approaches better mimicking in vivo conditions might reveal novel concepts of host interaction, also mediated by backbone subunits of Grampositive pili.

A common class of host target structures of many bacterial adhesins are glycoconjugates. Work by Day et al. (2017) describe P1 subunits as new pneumococcal lectins binding several 
glycosylation pattern: major P1 adhesin RrgA shows the broadest glycan binding repertoire (namely maltose, cellobiose, $\alpha / \beta$ linked galactose, blood group $\mathrm{A}$ and $\mathrm{H}$ antigens) when compared to $\mathrm{RrgB}$ and $\mathrm{RrgC}$ specificities. Interestingly, screening glycosylation targets of P1 subunits also identified non-human and nonmammalian patterns, assigning an even larger spectrum of potential P1 interactions (Day et al., 2017).

Apart from its potential role as lectin (Day et al., 2017), no further host interaction factor for $\mathrm{P} 1$ anchor $\mathrm{RrgC}$ is known (Table 1C).

The multiplicity of described P1-mediated host interaction scenarios makes P1 a pneumococcal virulence factor with strong impact on the pathogenesis of $S$. pneumoniae. P1 was shown to contribute to initial steps of colonization but also promotes invasion and spreading within the host (Table 1). Despite obvious advantages, P1 was identified in only a relatively small proportion of pneumococcal clinical isolates $(-30 \%)$ belonging to few clonal complexes (Dzaraly et al., 2020) and PI-1 positive strains show a biphasic P1 expression pattern (Angelis et al., 2011). This implies potential limitations for pneumococcal fitness and virulence related to P1 expression: synthesis of high molecular weight architecture of P1 is complex (Figure 1) and might be tightly regulated by the bacterial metabolism. Iovino et al. (2020) propose two trade-off mechanisms explaining the relatively low \% of P1 expressing strains: a high P1 induced host immunogenicity related to surface exposed P1 filaments that may also prevent recolonization and a low host transmissibility due to P1 adhesive properties. Additionally S. pneumoniae produces a variety of surface exposed, virulence related factors promoting various host interactions (Mitchell and Mitchell, 2010; Hilleringmann et al., 2015) that may substitute similar P1 mediated functions (e.g., PavA - fibronectin recognition). Importantly epidemiological data show that strain isolates that contain PI-1 are often associated with successful pneumococcal lineages and antimicrobial resistance pattern (Dzaraly et al., 2020), suggesting P1 as a particular tool among the diversity of pneumococcal virulence factors.

\section{FUTURE DIRECTION AND CONCLUDING REMARKS}

One and a half decades after the first description of S. pneumoniae P1 a considerable amount of data characterizes these very long, thin and highly stable surface appendages as evolutionary optimized subunit assemblies that promote pneumococcal virulence mediating multifunctional interactions in different host niches. Although the main components essential for P1

\section{REFERENCES}

Amerighi, F., Valeri, M., Donnarumma, D., Maccari, S., Moschioni, M., Taddei, A., et al. (2016). Identification of a monoclonal antibody against pneumococcal pilus 1 ancillary protein impairing bacterial adhesion to human epithelial cells. J. Infect. Dis. 213, 516-522. doi: 10.1093/infdis/ jiv461 formation and the resulting architecture are well described, details of the spatio-temporal interplay during P1 assembly on the bacterial surface and their regulation ("P1-assembly machinery") needs further analysis, applying, e.g., novel high resolution microscopic approaches. In addition, a more complete picture of $\mathrm{P} 1$ functional aspects and the in vivo relevance demands a greater focus on complementary experimental approaches mimicking host environments [e.g., mechanical force- conditions (Dufrêne and Persat, 2020; Viela et al., 2020)] in addition to suitable in vivo models. These data will allow a more profound evaluation of P1 mediated bacterial fitness benefits with related costs of P1 expression and the risk of P1 induced adverse host immune responses. Promising antigenic properties of P1 subunits were demonstrated in several animal studies (Gianfaldoni et al., 2007; Moschioni et al., 2010; Gentile et al., 2011; Harfouche et al., 2012). Although P1 is found in only 30\% of clinical pneumococcal isolates, its complex involvement with bacterial virulence, and data implying an association of $\mathrm{P} 1$ with antibiotic resistance and evolving non-vaccine serotypes (Dzaraly et al., 2020) make P1 subunits interesting protein-based vaccine candidates. Rational antigen (AG) design and innovative formulation strategies might enable a future non-serotype-dependent, efficient vaccination against $S$. pneumoniae, containing several protective pneumococcal protein-AGs, potentially in combination with known polysaccharide-conjugate AG designs (Converso et al., 2020). A direct interference with P1 function, like small molecule or antibody-mediated inhibition of PI-1 sortase activity, blocking of specific P1 epitopes involved in host interaction (Amerighi et al., 2016) or destabilizing P1 backbone subunits to reduce P1 functionality in host environment as shown for Spy0128, the major pilin from the Gram-positive human pathogen S. pyogenes (Rivas-Pardo et al., 2018) constitute further potential strategies to reduce P1-mediated virulence. In addition, understanding P1 on a molecular level also enabled the design of innovative bioconjugation tools (Bonnet et al., 2017) and demonstrates the many facets of this fascinating structure.

\section{AUTHOR CONTRIBUTIONS}

$\mathrm{SN}$ and $\mathrm{MH}$ wrote the manuscript and performed critical revision of the work. Both authors approved the submitted version.

\section{FUNDING}

This work was financially supported through the Open Access Publication fund of the Munich University of Applied Sciences (MUAS). 
Baker, E. N., Squire, C. J., and Young, P. G. (2015). Self-generated covalent crosslinks in the cell-surface adhesins of Gram-positive bacteria. Biochem. Soc. Trans. 43, 787-794. doi: 10.1042/bst20150066

Barocchi, M. A., Ries, J., Zogaj, X., Hemsley, C., Albiger, B., Kanth, A., et al. (2006). A pneumococcal pilus influences virulence and host inflammatory responses. Proc. Natl. Acad. Sci. U.S.A. 103, 2857-2862. doi: 10.1073/pnas.0511017103

Basset, A., Herd, M., Daly, R., Dove, S. L., and Malley, R. (2017). The Pneumococcal type 1 Pilus genes are thermoregulated and are repressed by a member of the snf2 protein family. J. Bacteriol. 199:e00078-17.

Basset, A., Turner, K. H., Boush, E., Sayeed, S., Dove, S. L., and Malley, R. (2011). Expression of the type 1 pneumococcal pilus is bistable and negatively regulated by the structural component RrgA. Infect. Immun. 79, 2974-2983. doi: 10.1128/ iai.05117-11

Basset, A., Turner, K. H., Boush, E., Sayeed, S., Dove, S. L., and Malley, R. (2012). An epigenetic switch mediates bistable expression of the type 1 pilus genes in Streptococcus pneumoniae. J. Bacteriol. 194, 1088-1091. doi: 10.1128/jb. 06078-11

Basset, A., Zhang, F., Benes, C., Sayeed, S., Herd, M., Thompson, C., et al. (2013). Toll-like receptor (TLR) 2 mediates inflammatory responses to oligomerized RrgA pneumococcal pilus type 1 protein. J. Biol. Chem. 288, 2665-2675. doi: 10.1074/jbc.m112.398875

Becke, T. D., Ness, S., Gürster, R., Schilling, A. F., Di Guilmi, A.-M., Sudhop, S., et al. (2018). Single molecule force spectroscopy reveals two-domain binding mode of pilus-1 tip protein RrgA of Streptococcus pneumoniae to Fibronectin. ACS Nano 12, 549-558. doi: 10.1021/acsnano.7b07247

Becke, T. D., Ness, S., Kaufmann, B. K., Hartmann, B., Schilling, A. F., Sudhop, S., et al. (2019). Pilus-1 backbone protein RrgB of Streptococcus pneumoniae binds collagen I in a force-dependent way. ACS Nano 13, 7155-7165. doi: 10.1021/acsnano. $9 \mathrm{~b} 02587$

Binsker, U., Lees, J. A., Hammond, A. J., and Weiser, J. N. (2020). Immune exclusion by naturally acquired secretory IgA against pneumococcal pilus-1. J. Clin. Invest. 130, 927-941. doi: 10.1172/jci132005

Bonnet, J., Cartannaz, J., Tourcier, G., Contreras-Martel, C., Kleman, J. P., Morlot, C., et al. (2017). Autocatalytic association of proteins by covalent bond formation: a Bio Molecular Welding toolbox derived from a bacterial adhesin. Sci. Rep. 7:43564.

Chang, C., Wu, C., Osipiuk, J., Siegel, S. D., Zhu, S., Liu, X., et al. (2019). Cell-tocell interaction requires optimal positioning of a pilus tip adhesin modulated by gram-positive transpeptidase enzymes. Proc. Natl. Acad. Sci. U.S.A. 116, 18041-18049. doi: 10.1073/pnas.1907733116

Chen, Y.-H., Li, S.-H., Yang, Y.-C., Hsu, S.-H., Nizet, V., and Chang, Y.-C. (2020). T4 Pili promote colonization and immune evasion phenotypes of nonencapsulated M4 Streptococcus pyogenes. mBio 11:e01580-20.

Converso, T. R., Assoni, L., André, G. O., Darrieux, M., and Leite, L. C. C. (2020). The long search for a serotype independent pneumococcal vaccine. Expert Rev. Vaccines 19, 57-70. doi: 10.1080/14760584.2020.1711055

Day, C. J., Paton, A. W., Harvey, R. M., Hartley-Tassell, L. E., Seib, K. L., Tiralongo, J., et al. (2017). Lectin activity of the pneumococcal pilin proteins. Sci. Rep. 7:17784.

Dufrêne, Y. F., and Persat, A. (2020). Mechanomicrobiology: how bacteria sense and respond to forces. Nat. Rev. Microbiol. 18, 227-240. doi: 10.1038/s41579019-0314-2

Dzaraly, N. D., Muthanna, A., Mohd Desa, M. N., Taib, N. M., Masri, S. N., Rahman, N. I. A., et al. (2020). Pilus islets and the clonal spread of piliated Streptococcus pneumoniae: a review. Int. J. Med. Microbiol. 310:151449. doi: 10.1016/j.ijmm.2020.151449

Echelman, D. J., Alegre-Cebollada, J., Badilla, C. L., Chang, C., Ton-That, H., and Fernández, J. M. (2016). CnaA domains in bacterial pili are efficient dissipaters of large mechanical shocks. Proc. Natl. Acad. Sci. U.S.A. 113, 2490-2495. doi: 10.1073/pnas.1522946113

El Mortaji, L., Contreras-Martel, C., Moschioni, M., Ferlenghi, I., Manzano, C., Vernet, T., et al. (2012a). The full-length Streptococcus pneumoniae major pilin $\operatorname{RrgB}$ crystallizes in a fibre-like structure, which presents the D1 isopeptide bond and provides details on the mechanism of pilus polymerization. Biochem. J. 441, 833-841. doi: 10.1042/bj20111397

El Mortaji, L., Fenel, D., Vernet, T., and Di Guilmi, A. M. (2012b). Association of $\operatorname{RrgA}$ and $\mathrm{RrgC}$ into the Streptococcus pneumoniae pilus by sortases C-2 and C-3. Biochemistry 51, 342-352. doi: 10.1021/bi201591n
El Mortaji, L., Terrasse, R., Dessen, A., Vernet, T., and Di Guilmi, A. M. (2010). Stability and assembly of pilus subunits of Streptococcus pneumoniae. J. Biol. Chem. 285, 12405-12415. doi: 10.1074/jbc.m109.082776

Fälker, S., Nelson, A. L., Morfeldt, E., Jonas, K., Hultenby, K., Ries, J., et al. (2008). Sortase-mediated assembly and surface topology of adhesive pneumococcal pili. Mol. Microbiol. 70, 595-607. doi: 10.1111/j.1365-2958.2008.06396.x

Figueira, M., Moschioni, M., Angelis, G., de, Barocchi, M., Sabharwal, V., et al. (2014). Variation of pneumococcal Pilus-1 expression results in vaccine escape during Experimental Otitis Media EOM. PLoS One 9:e83798. doi: 10.1371/ journal.pone. 0083798

Gentile, M. A., Melchiorre, S., Emolo, C., Moschioni, M., Gianfaldoni, C., Pancotto, L., et al. (2011). Structural and functional characterization of the Streptococcus pneumoniae RrgB pilus backbone D1 domain. J. Biol. Chem. 286, 14588-14597. doi: 10.1074/jbc.m110.202739

Gianfaldoni, C., Censini, S., Hilleringmann, M., Moschioni, M., Facciotti, C., Pansegrau, W., et al. (2007). Streptococcus pneumoniae pilus subunits protect mice against lethal challenge. Infect. Immun. 75, 1059-1062. doi: 10.1128/iai. 01400-06

Gómez-Mejia, A., Gámez, G., Hirschmann, S., Kluger, V., Rath, H., Böhm, S., et al. (2018). Pneumococcal metabolic adaptation and colonization are regulated by the two-component regulatory system 08 . mSphere 3:e0165-18.

Harfouche, C., Filippini, S., Gianfaldoni, C., Ruggiero, P., Moschioni, M., Maccari, S., et al. (2012). RrgB321, a fusion protein of the three variants of the pneumococcal pilus backbone $\operatorname{RrgB}$, is protective in vivo and elicits opsonic antibodies. Infect. Immun. 80, 451-460. doi: 10.1128/iai.05780-11

Hava, D. L., LeMieux, J., and Camilli, A. (2003). From nose to lung: the regulation behind Streptococcus pneumoniae virulence factors. Mol. Microbiol. 50, 11031110. doi: 10.1046/j.1365-2958.2003.03764.x

Hendrickx, A. P. A., Budzik, J. M., Oh, S.-Y., and Schneewind, O. (2011). Architects at the bacterial surface - sortases and the assembly of pili with isopeptide bonds. Nat. Rev. Microbiol. 9, 166-176. doi: 10.1038/nrmicro2520

Herbert, J. A., Mitchell, A. M., and Mitchell, T. J. (2015). A serine-threonine kinase (StkP) regulates expression of the Pneumococcal pilus and modulates bacterial adherence to human epithelial and endothelial cells in vitro. PLoS One 10:e0127212. doi: 10.1371/journal.pone.0127212

Herman-Bausier, P., Labate, C., Towell, A. M., Derclaye, S., Geoghegan, J. A., and Dufrêne, Y. F. (2018). Staphylococcus aureus clumping factor A is a forcesensitive molecular switch that activates bacterial adhesion. Proc. Natl. Acad. Sci. U.S.A. 115, 5564-5569. doi: 10.1073/pnas.1718104115

Hilleringmann, M., Giusti, F., Baudner, B. C., Masignani, V., Covacci, A., Rappuoli, R., et al. (2008). Pneumococcal pili are composed of protofilaments exposing adhesive clusters of Rrg A. PLoS Pathog. 4:e1000026. doi: 10.1371/journal.ppat. 1000026

Hilleringmann, M., Kohler, S., Gámez, G., and Hammerschmidt, S. (2015). "Pneumococcal pili and adhesins," in Streptococcus pneumoniae Molecular Mechanisms of Host-Pathogen Interactions. eds J. Brown, S. Hammerschmidt and C. Orihuela (London: Elsevier).

Hilleringmann, M., Ringler, P., Müller, S. A., Angelis, G., de, Rappuoli, R., et al. (2009). Molecular architecture of Streptococcus pneumoniae TIGR4 pili. EMBO J. 28, 3921-3930. doi: 10.1038/emboj.2009.360

Hospenthal, M. K., Costa, T. R. D., and Waksman, G. (2017). A comprehensive guide to pilus biogenesis in Gram-negative bacteria. Nat. Rev. Microbiol. 15, 365-379. doi: 10.1038/nrmicro.2017.40

Iovino, F., Engelen-Lee, J.-Y., Brouwer, M., van de Beek, D., van der Ende, A., Valls Seron, M., et al. (2017). pIgR and PECAM-1 bind to pneumococcal adhesins RrgA and PspC mediating bacterial brain invasion. J. Exp. Med. 214, 1619-1630. doi: $10.1084 /$ jem. 20161668

Iovino, F., Hammarlöf, D. L., Garriss, G., Brovall, S., Nannapaneni, P., and Henriques-Normark, B. (2016a). Pneumococcal meningitis is promoted by single cocci expressing pilus adhesin RrgA. J. Clin. Invest. 126, 2821-2826. doi: $10.1172 /$ jci 84705

Iovino, F., Nannapaneni, P., Henriques-Normark, B., and Normark, S. (2020). The impact of the ancillary pilus-1 protein RrgA of Streptococcus pneumoniae on colonization and disease. Mol. Microbiol. 113, 650-658. doi: 10.1111/mmi. 14451

Iovino, F., Seinen, J., Henriques-Normark, B., and van Dijl, J. M. (2016b). How Does Streptococcus pneumoniae invade the brain? Trends Microbiol. 24, 307315. doi: $10.1016 /$ j.tim.2015.12.012 
Izoré, T., Contreras-Martel, C., El Mortaji, L., Manzano, C., Terrasse, R., Vernet, T., et al. (2010). [Duplikat] Structural basis of host cell recognition by the pilus adhesin from Streptococcus pneumoniae. Structure 18, 106-115. doi: 10.1016/j. str.2009.10.019

Jacobitz, A. W., Naziga, E. B., Yi, S. W., McConnell, S. A., Peterson, R., Jung, M. E., et al. (2016). The "Lid" in the Streptococcus pneumoniae SrtC1 sortase adopts a rigid structure that regulates substrate access to the active site. J. Phys. Chem. B 120, 8302-8312. doi: 10.1021/acs.jpcb.6b01930

Kang, H. J., Coulibaly, F., Clow, F., Proft, T., and Baker, E. N. (2007). Stabilizing isopeptide bonds revealed in gram-positive bacterial pilus structure. Science 318, 1625-1628. doi: 10.1126/science.1145806

Khare, B., and Narayana, S. V. L. (2017). Pilus biogenesis of Gram-positive bacteria: roles of sortases and implications for assembly. Protein Sci. 26, 1458-1473. doi: 10.1002/pro.3191

Kreikemeyer, B., Gámez, G., Margarit, I., Giard, J.-C., Hammerschmidt, S., Hartke, A., et al. (2011). Genomic organization, structure, regulation and pathogenic role of pilus constituents in major pathogenic Streptococci and Enterococci. Int. J. Med. Microbiol. 301, 240-251. doi: 10.1016/j.ijmm.2010.09.003

Krishnan, V., Gaspar, A. H., Ye, N., Mandlik, A., Ton-That, H., and Narayana, S. V. L. (2007). An IgG-like domain in the minor pilin GBS52 of Streptococcus agalactiae mediates lung epithelial cell adhesion. Structure 15, 893-903. doi: 10.1016/j.str.2007.06.015

Laurenceau, R., Péhau-Arnaudet, G., Baconnais, S., Gault, J., Malosse, C., Dujeancourt, A., et al. (2013). A type IV pilus mediates DNA binding during natural transformation in Streptococcus pneumoniae. PLoS Pathog. 9:e1003473. doi: 10.1371/journal.ppat.1003473

LeMieux, J., Hava, D. L., Basset, A., and Camilli, A. (2006). RrgA and RrgB are components of a multisubunit pilus encoded by the Streptococcus pneumoniae rlrA pathogenicity islet. Infect. Immun. 74, 2453-2456. doi: 10.1128/iai.74.4. 2453-2456.2006

LeMieux, J., Woody, S., and Camilli, A. (2008). Roles of the sortases of Streptococcus pneumoniae in assembly of the RlrA pilus. J. Bacteriol. 190, 6002-6013. doi: 10.1128/jb.00379-08

Loughran, A. J., Orihuela, C. J., and Tuomanen, E. I. (2019). Streptococcus pneumoniae: invasion and inflammation. Microbiol. Spectr. 7:10.1128/microbiolspec.GPP3-0004-2018.

Mandlik, A., Das, A., and Ton-That, H. (2008). The molecular switch that activates the cell wall anchoring step of pilus assembly in gram-positive bacteria. Proc. Natl. Acad. Sci. U.S.A. 105, 14147-14152. doi: 10.1073/pnas.0806350105

Manzano, C., Contreras-Martel, C., El Mortaji, L., Izoré, T., Fenel, D., Vernet, T., et al. (2008). Sortase-mediated pilus fiber biogenesis in Streptococcus pneumoniae. Structure 16, 1838-1848. doi: 10.1016/j.str.2008.10.007

Manzano, C., Izoré, T., Job, V., Di Guilmi, A. M., and Dessen, A. (2009). Sortase activity is controlled by a flexible lid in the pilus biogenesis mechanism of grampositive pathogens. Biochemistry 48, 10549-10557. doi: 10.1021/bi901261y

Mitchell, A. M., and Mitchell, T. J. (2010). Streptococcus pneumoniae: virulence factors and variation. Clin. Microbiol. Infect. 16, 411-418. doi: 10.1111/j.14690691.2010.03183.x

Moschioni, M., Emolo, C., Biagini, M., Maccari, S., Pansegrau, W., Donati, C., et al. (2010). The two variants of the Streptococcus pneumoniae pilus $1 \mathrm{RrgA}$ adhesin retain the same function and elicit cross-protection in vivo. Infect. Immun. 78, 5033-5042. doi: 10.1128/iai.00601-10

Muhammad, M. H., Idris, A. L., Fan, X., Guo, Y., Yu, Y., Jin, X., et al. (2020). Beyond risk: bacterial biofilms and their regulating approaches. Front. Microbiol. 11:928. doi: 10.3389/fmicb.2020.00928

Muñoz-Elías, E. J., Marcano, J., and Camilli, A. (2008). Isolation of Streptococcus pneumoniae biofilm mutants and their characterization during nasopharyngeal colonization. Infect. Immun. 76, 5049-5061. doi: 10.1128/iai.00425-08

Muschiol, S., Aschtgen, M.-S., Nannapaneni, P., and Henriques-Normark, B. (2019). Gram-positive type IV pili and competence. Microbiol. Spectr. 7, 129-135.

Naziga, E. B., and Wereszczynski, J. (2017). Molecular mechanisms of the binding and specificity of Streptococcus pneumoniae Sortase C enzymes for pilin subunits. Sci. Rep. 7:13119.

Neiers, F., Madhurantakam, C., Fälker, S., Manzano, C., Dessen, A., Normark, S., et al. (2009a). Two crystal structures of pneumococcal pilus sortase C provide novel insights into catalysis and substrate specificity. J. Mol. Biol. 393, 704-716. doi: $10.1016 /$ j.jmb.2009.08.058
Neiers, F., Madhurantakam, C., Fälker, S., Normark, S., Henriques-Normark, B., and Achour, A. (2009b). Cloning, expression, purification, crystallization and preliminary X-ray analysis of the pilus-associated sortase C from Streptococcus pneumoniae. Acta Crystallogr. Sect. F Struct. Biol. Cryst. Commun. 65, 55-58. doi: 10.1107/s1744309108040025

Nelson, A. L., Ries, J., Bagnoli, F., Dahlberg, S., Fälker, S., Rounioja, S., et al. (2007). RrgA is a pilus-associated adhesin in Streptococcus pneumoniae. Mol. Microbiol. 66, 329-340. doi: 10.1111/j.1365-2958.2007.05908.x

Orrskog, S., Rounioja, S., Spadafina, T., Gallotta, M., Norman, M., Hentrich, K., et al. (2012). Pilus adhesin RrgA interacts with complement receptor 3, thereby affecting macrophage function and systemic pneumococcal disease. mBio 4:e00535-12.

Pancotto, L., Angelis, G., de, Bizzarri, E., Barocchi, M. A., Del Giudice, G., et al. (2013). Expression of the Streptococcus pneumoniae pilus-1 undergoes on and off switching during colonization in mice. Sci. Rep. 3:2040.

Paterson, N. G., and Baker, E. N. (2011). Structure of the full-length major pilin from Streptococcus pneumoniae: implications for isopeptide bond formation in gram-positive bacterial pili. PLoS One 6:e22095. doi: 10.1371/journal.pone. 0022095

Ramirez, N. A., Das, A., and Ton-That, H. (2020). New paradigms of pilus assembly mechanisms in gram-positive Actinobacteria. Trends Microbiol. 28, 999-1009. doi: 10.1016/j.tim.2020.05.008

Rivas-Pardo, J. A., Badilla, C. L., Tapia-Rojo, R., Alonso-Caballero, Á., and Fernández, J. M. (2018). Molecular strategy for blocking isopeptide bond formation in nascent pilin proteins. Proc. Natl. Acad. Sci. U.S.A. 115, 92229227. doi: 10.1073/pnas.1807689115

Shaik, M. M., Maccagni, A., Tourcier, G., Di Guilmi, A. M., and Dessen, A. (2014). Structural basis of pilus anchoring by the ancillary pilin RrgC of Streptococcus pneumoniae. J. Biol. Chem. 289, 16988-16997. doi: 10.1074/jbc.m114. 555854

Spirig, T., Weiner, E. M., and Clubb, R. T. (2011). Sortase enzymes in Grampositive bacteria. Mol. Microbiol. 82, 1044-1059. doi: 10.1111/j.1365-2958. 2011.07887.x

Spraggon, G., Koesema, E., Scarselli, M., Malito, E., Biagini, M., Norais, N., et al. (2010). Supramolecular organization of the repetitive backbone unit of the Streptococcus pneumoniae pilus. PLoS One 5:e10919. doi: 10.1371/journal.pone. 0010919

Subramanian, K., Henriques-Normark, B., and Normark, S. (2019). Emerging concepts in the pathogenesis of the Streptococcus pneumoniae: from nasopharyngeal colonizer to intracellular pathogen. Cell. Microbiol. 21:e13077.

Tabusi, M., Thorsdottir, S., Lysandrou, M., Narciso, A. R., Minoia, M., HenriquesNormark, B., et al. (2020). Neuronal death in pneumococcal meningitis is triggered by pneumolysin and pilus- 1 interactions with $\beta$-actin. bioRxiv [Preprint]. doi: 10.1101/2020.08.20.258681

Telford, J. L., Barocchi, M. A., Margarit, I., Rappuoli, R., and Grandi, G. (2006). Pili in gram-positive pathogens. Nat. Rev. Microbiol. 4, 509-519.

Tsai, J.-Y. C., Loh, J. M. S., Clow, F., Lorenz, N., and Proft, T. (2017). The Group A Streptococcus serotype M2 pilus plays a role in host cell adhesion and immune evasion. Mol. Microbiol. 103, 282-298. doi: 10.1111/mmi.13556

Viela, F., Mathelié-Guinlet, M., Viljoen, A., and Dufrêne, Y. F. (2020). What makes bacterial pathogens so sticky? Mol. Microbiol. 113, 683-690. doi: 10.1111/mmi. 14448

Young, P. G., Moreland, N. J., Loh, J. M., Bell, A., Atatoa Carr, P., Proft, T., et al. (2014). Structural conservation, variability, and immunogenicity of the T6 backbone pilin of serotype M6 Streptococcus pyogenes. Infect. Immun. 82, 2949-2957.

Conflict of Interest: The authors declare that the research was conducted in the absence of any commercial or financial relationships that could be construed as a potential conflict of interest.

Copyright (C) 2021 Ness and Hilleringmann. This is an open-access article distributed under the terms of the Creative Commons Attribution License (CC BY). The use, distribution or reproduction in other forums is permitted, provided the original author(s) and the copyright owner(s) are credited and that the original publication in this journal is cited, in accordance with accepted academic practice. No use, distribution or reproduction is permitted which does not comply with these terms. 